\title{
TOWARDS A MODEL OF ARGUMENT STRENGTH FOR BIPOLAR ARGUMENTATION GRAPHS
}

\begin{abstract}
Bipolar argument graphs represent the structure of complex pro and contra arguments for one or more standpoints. In this article, ampliative and exclusionary principles of evaluating argument strength in bipolar acyclic argumentation graphs are laid out and compared to each other. Argument chains, linked arguments, link attackers and supporters, and convergent arguments are discussed. The strength of conductive arguments is also addressed but it is argued that more work on this type of argument is needed to properly distinguish argument strength from more general value-based components of such arguments. The overall conclusion of the article is that there is no justifiably unique solution to the problem of argument strength outside of a particular epistemological framework.

Keywords: argument structure, conductive argument, convergent argument, possibility theory, argument visualization.
\end{abstract}

\section{Introduction}

The goal of this article is to lay out minimal conditions for the way in which the strength of arguments is combined into an overall strength of a more complex argument on the basis of an initial intuitive assessment of the strength of its subarguments. Although the principles in this article are based on existing research, there is no agreement about how to deal with argument strength in the seminal literature, because the ways in which argument strength may be computed depend on the presumed theory of argument structure. Authors have proposed different ways of structuring arguments and slightly different taxonomies of types of argument structure. I, therefore, take a rather permissive and broad stance about argument structure in this article and attempt to determine intuitively appealing principles for the combination of argument strength for those argument structure types that have been proposed. This does not indicate that I endorse or recommend the given taxonomy, which will to some extent always 
remain arbitrary. The focus of this article is on finding general principles for the combination of argument strength. If someone believes that a certain type of argument structure, such as 'conductive argument', does not exist, no harm is done by ignoring the corresponding principles. The discussion of the principles is deliberately condensed for reasons of space, but in order to properly situate the account within the literature on argument strength a number of basic distinctions are needed. These are laid out in the remainder of this introductory section.

In positional accounts of argument strength, arguments are evaluated with respect to their place in a larger argumentation network in which they occur, but often no degree of strength is assigned to individual premises or conclusions. In Dung's Abstract Argumentation Frameworks (AAFs) (Dung 1995), for instance, argumentation is modelled on the basis of attack relations between arguments, and arguments are either "in" or "out" depending on whether they are successfully attacked by other arguments or not. In his original framework Dung only defines an attack relation, and support only exists in a derived way based on the notion of defending an argument against an attack. An argument $a$ supports another argument $b$ in this theory only if $a$ attacks an argument $c$ that attacks $b$. This way of modelling support has been criticized by Cayrol \& Lagasquie-Schiex (2005), who argue that attacking an attacker is not the same as supporting an argument directly, and therefore extended AAFs into bipolar argumentation frameworks (BAFs). These consist of a set of arguments, a relation of support, and an attack relation between them (Cayrol \& LagasquieSchiex, 2009, 66-68). They also consider assigning initial values to arguments in a BAF and ways to extend these to a full valuation of the network on the basis of whether they are attacked with some strength or defeated. These accounts utilize a notion of graded argument strength that is also the topic of this article. However, their approach does not do justice to more general work on argument structure, because they do not distinguish between linked arguments and convergent arguments and between convergent and conductive arguments. The first distinction was introduced by Thomas (1977) and is explicitly taken into account by Yanal (1991), Walton (1996), Freeman (2011), and Selinger (2014) among others. The term 'conductive argument' is in turn used by Wellman (1971), Govier (2013), Zenker (2011) and others, although there is no general agreement on a definition and terminology differs slightly from author to author. ${ }^{1}$

Unfortunately, even in bipolar graded approaches that deal with both linked and convergent arguments, there is no agreement about the key principles that a measure of argument strength must satisfy. On the one hand, 
there are probabilistic approaches such as Nielsen \& Parsons (2006) and to some extent Yanal (1991) that take the probabilities that the premises of an inference are true as a basis for an evaluation of a larger chain of inference. On the other hand, others such as Selinger (2014) are interested in the credibility or acceptability of arguments, where the rules governing acceptability need not necessarily follow the probability calculus and rules for knowledge transmission in Bayesian networks. One may call these acceptability-based.

Another important distinction is that between ampliative, nonampliative, and exclusionary theories of argument strength. These are based on a distinction between the quantity of arguments versus their quality. Argument quantity is defined as the number of arguments in a complex argumentation that can independently support a conclusion. In contrast to this, the quality of an argument is given by its soundness, validity, and other standards of correctness of the premises and the way in which these are supposed to support the conclusion. In an ampliative or cumulative approach like that of Cayrol \& Lagasquie-Schiex (2009) and Selinger (2014), multiple independent arguments for the same standpoint generally increase the strength of the overall argumentation. In other words, quantity influences quality in these accounts. In contrast to this, in a nonampliative account the overall strength of the argumentation does not increase by adding additional, redundant inferences to the same conclusion. As a special case of the nonampliative approach, in an exclusionary account there is no functional relation between argument quantity and quality.

With respect to these distinctions, the account in this article is bipolar, graded, and acceptability-based. Both exclusionary and ampliative principles will be discussed, and the account also gives justice to the distinction between linked and convergent arguments. However, the goal of this article is not to generally contrast this type of approach with the others mentioned above, but rather to identify a small set of rationality principles from which more specific theories of argument strength can be derived.

\section{Bipolar Argumentation Graphs}

\subsection{Argument Structure}

In this section I introduce bipolar argumentation graph (BAG) structures. Since none of the postulates and rules to be discussed hinges on particular features of a particular representation of argument structure (nor should they), it suffices to lay out a number of basic subgraphs out of which larger graphs can be composed. These are annotated with one or more 
premises at nodes, a conclusion, inference links, the polarity of a link as $\oplus$ for pro and $\ominus$ for contra, and an initial assessment of argument strength, which is attached to the premises in linked and conductive arguments and to the link in the basic part of a convergent argument.

Here is the list of admissible subgraphs:

- Basic arguments: A basic argument consists of a pro or contra inference link from a single premise to a conclusion. A basic argument is depicted as an arrow from a premise to a conclusion, labelled with its polarity and an initial assessment of its strength.

- Convergent arguments (Figure 1): These consist of several basic, linked, or conductive arguments towards the same conclusion, where all links must be either pro or contra. They are depicted by multiple arrows that lead to a conclusion. The arrows are marked with possibly different strengths, but the same polarity, for all basic arguments of a convergent argument.

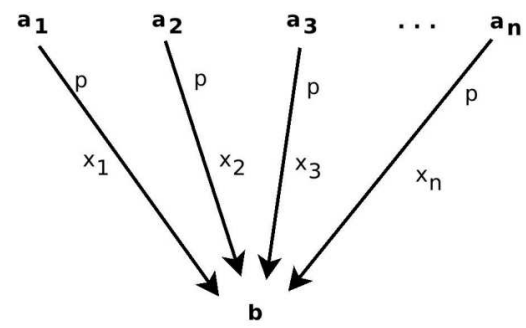

Figure 1. Structure of a convergent argument with initial strengths $x_{1}, \ldots, x_{n}$ and polarity $p$. Each arrow constitutes a basic argument for standpoint $b$, but the argument as a whole forms a cohesive unit

- Linked arguments (Figure 2): These link several premises together to support or attack a conclusion. They are depicted by a horizontal line from which an arrow leads to a conclusion, where the arrow is labelled with a polarity. As I will lay out in more detail below, an initial assessment of strength of a linked argument is based on individual strengths of each premise with respect to the argument as a whole.

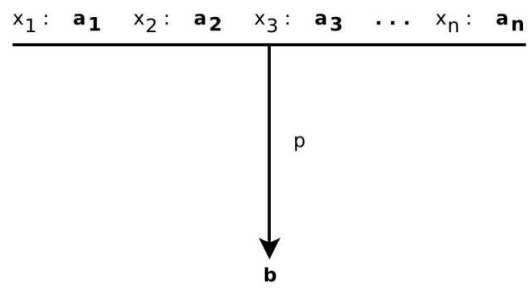

Figure 2. Structure of a linked argument with initial strengths $x_{1}, \ldots, x_{n}$ and polarity $p$. Removing a link will result in a much less conclusive or an inconclusive argument 
- Conductive arguments (Figure 3): These link several premises together to support or attack a conclusion on the basis of trade-off considerations. They are depicted like linked arguments but with a double arrow. As in linked arguments, an initial assessment of their strength depends on the individual strengths of their premises. In addition to linked arguments, however, individual premises are marked with polarities. As a further constraint, if a link has polarity $p$, then at least one premise must have polarity $p$ as well.

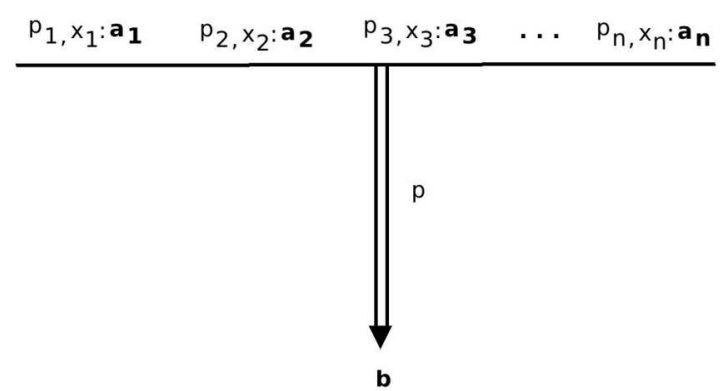

Figure 3. Structure of a conductive argument with initial strengths/values $x_{1}, \ldots$, $x_{n}$, individual polarities $p_{1}, \ldots, p_{n}$ and overall polarity $p$. Additional evaluations $v_{1}\left(a_{1}\right), v_{2}\left(a_{2}\right), \ldots, v_{n}\left(a_{n}\right)$ will be needed

- Link attackers and link supporters (Figure 4): These support or attack the individual inference links rather than premises. They are depicted as arrows to the middle of an arrow and annotated with polarity and an initial assessment of their strength like the other types of argument. The original premises can be linked, conductive, or part of a basic argument, and the attacking argument $(C$, in the figure) may be of any type, including a convergent argument with several attack or support links.

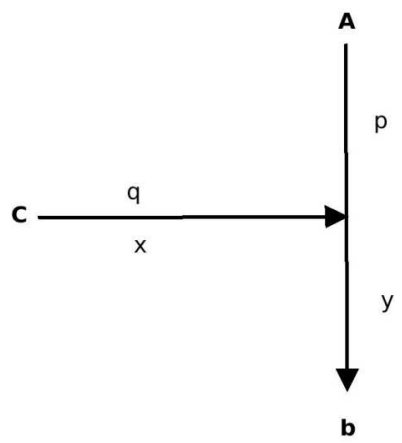

Figure 4. An argument for or against the inference link of another argument with strength $x$ and polarity $q ; A$ and $C$ can have a complex argument structure and the inference from $A$ to $b$ can be of various structure types 
A conclusion can itself form a premise of any of the above argument types. Since BAGs are also finite and acyclic (more on this below), they have initial nodes and terminal nodes. If there is an inference link (arrow) from $A$ to $B$, then $A$ is the antecedent of $B$ and $B$ the successor of $A$. Sometimes also an argument as a whole will be called an antecedent if an arrow leads away from its conclusion; whether an individual premise or an argument as a whole is meant will be clear from the context. Initial nodes are those nodes that contain premises without antecedents and terminal nodes are those nodes that contain conclusions without successors.

We may speak of an argument chain to a conclusion $b$ whenever there are two or more arrows from premises $A_{1}, \ldots, A_{n}$ that lead to $b$ on a direct path: $A_{1} \rightarrow A_{2} \rightarrow \ldots \rightarrow A_{n} \rightarrow B$. The links and premises can form any type of argument subgraph, and so capital letters are used to distinguish them from individual premises. Chains of two arguments are distinguished into pro-pro, pro-contra, contra-pro, and contra-contra chains according to the order of polarity of the inference links in the chain from antecedents to successors (see Figure 5).

(a)

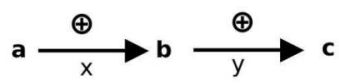

(b)

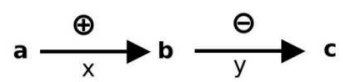

(c)

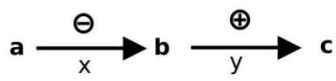

(d)

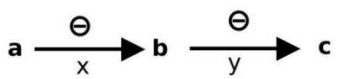

Figure 5. Four types of direct chains: (a) pro-pro, (b) pro-contra, (c) contra-pro, and $(d)$ contra-contra chain

Among these, pro-contra and contra-pro are mixed chains. A chain starting with a pro (contra) argument is called a pro (contra) chain. Correspondingly, a bundle on $b$ comprises all the inference links with antecedent premises and their annotations that have $b$ as their conclusion, and bundles can be pro, contra, or mixed. Pro bundles may constitute convergent pro arguments and contra bundles convergent contra arguments, but they do not need to. If they are deemed not to have enough thematic unity, then they might also be classified as multiple argumentation, which is laid out in Section 3.6. Bundles with mixed polarity are always cases of multiple argumentation.

Three more requirements are needed. The graphs also have to be $f$ nite, acyclic, and connected. In contrast to positional accounts like AAFs and BAFs, which allow for cycles, acyclicity is needed in the present approach for a proper recursive assessment of argument strength. The additional requirements of connectedness and finiteness are trivial. They are 
finite, because human beings and machines can only consider finitely many arguments at a time. Regarding connectedness, if there were several disconnected subgraphs, then we could evaluate argument strength in each of them separately, since there is no global interaction between them in our approach. Hence, connected graphs can be assumed without loss of generality. $^{2}$

\subsection{Remarks}

The graphs introduced above capture the main types of argument structure that are commonly found in the literature. Linked and convergent arguments have been discussed under various names, see Freeman (2011, Ch. 1) for an overview. Conductive arguments go back to Wellman $(1971,1975)$. Since then, some authors such as Hitchcock $(1980,1983)$ and contributions to Blair (2011) accept them, whereas others like Adler (2013) reject them. Entering this debate would go beyond the scope of this article.

A key assumption of the present approach to argument strength is that it is possible to somehow intuitively assess the strength of arguments in isolation first, and the goal is then to elaborate a theory of combinative argument strength on the basis of this initial assessment and suitable rationality postulates. Cayrol \& Lagasquie-Schiex $(2005,2009)$ and Selinger (2014) also make this assumption, among others.

\section{Combinative Argument Strength}

In this section, I lay out and justify general principles for determining the strength of complex arguments, i.e., principles for the computation of argument strength in chains and convergent arguments on the basis of the strength of their antecedents. In contrast to the initial assessment, I call this aggregation of strength for larger structures combinative argument strength.

\subsection{Basic Principles}

Cayrol \& Lagasquie-Schiex suggest three principles for BAFs that argument strength combination should obey, which they label P1-P3 (Cayrol \& Lagasquie-Schiex 2005: 78). They are quoted below, but with my own naming scheme.

\section{(DA) Direct Attack Principle}

"The value of an argument depends on the values of its direct attackers and of its direct supporters." (Cayrol \& Lagasquie-Schiex 2005: 78) 


\section{(BQ) Bipolar Quality Principle}

"If the quality of the support (resp. attack) increases then the value of the argument increases (resp. decreases)." (ibid.)

\section{(AP) Ampliativity}

"If the quantity of the supports (resp. attacks) increases then the quality of the support (resp. attack) increases." (ibid.)

Of these principles the first two are obvious, as they directly reflect what the words 'support' and 'attack' mean, for which the more neutral terms 'pro' and 'contra' are in my opinion preferable, as well as 'argument strength' instead of 'value' for reasons that will become more apparent in Section 3.7. The principles are necessary, though not sufficient adequacy requirements for a theory of argument strength in a bipolar setting. I disagree with Cayrol \& Lagasquie-Schiex, Selinger, and many others about ampliativity, though. Such an approach might capture some psychological effects, but ampliative argument strength cannot hold in general, because multiple premises for the same conclusion are redundant in a purely deductive setting and can lead to weaker conclusions when any kind of nonmonotonic inference pattern, such as abduction or induction, is used. This issue will be addressed again in Section 4. Since my aim is to find minimal requirements for an acceptability-based theory of argument strength, I therefore suggest the following principle instead of AP:

\section{(EN) Neutrality}

The number of pro (contra) arguments in a bundle on $a$ does not influence the argument strength of $a$.

Note that this principle holds for pro and contra bundles, but not necessarily for mixed ones. For the latter it could make sense to stipulate a separate principle that governs the way in which the strengths of pro and contra arguments determine the overall strength of a standpoint, but the exclusionary approach in Section 3.6 will not be mixed in that sense.

Principles DA, BQ, and EN are obviously not sufficient to determine modes of argument strength combination. In the following paragraphs, I will lay out more detailed principles and their justifications for the combination of argument strength in linked arguments, chains, bundles, and convergent arguments. 


\subsection{The Strength of Convergent Arguments}

The idea behind convergent arguments is that an arguer provides multiple supports of varying strength for or against some standpoint and that this support has some thematic unity that makes it reasonable to consider the whole discourse fragment as one argument, as opposed to thematically more disconnected multiple argumentation. Here is an example of a convergent argument for conclusion $c$ with two basic arguments $p_{1}$ and $p_{2}$ : "(c) It's going to rain tomorrow, $\left(p_{1}\right)$ [because] the weather forecast has predicted it and $\left(p_{2}\right)$ I know these kind of clouds: They bring rain."

\section{Exclusionary Approach}

The idea behind the following principle is that when several arguments speak for a certain standpoint an arguer could, in theory, pick only the strongest of them and discard all others, and because of the proposed independence of argument strength and polarity this also holds for contra bundles.

\section{(EP) Exclusionary Principle}

The combined strength of a pro (contra) convergent argument is the maximum of the strengths of its basic arguments.

So, it seems that in a theory with the Neutrality Principle this is the only reasonable choice. But, as I laid out at the beginning of this section, this is one of the most controversial issues, and only one of three different theories of argument strength: In an ampliative theory, the bundle strength is an increasing function of all pro (contra) links, in a nonampliative theory the bundle strength may not be higher than the maximum strength of pro (contra) links of the bundle, and in an exclusionary theory exactly the maximum is chosen. All of these principles hinge on the assumption that the inferences in a bundle are independent of each other with respect to the inference type--deductive, inductive, abductive, and so forth--, since otherwise specific rules of the inference type will govern their combination (see Section 4).

\section{Ampliative Approach}

The general idea behind convergent arguments in an approach that accepts AP is captured by the following principle:

\section{(ACU) Cumulative Principle}

The strength of convergent arguments consisting of $n>1$ independent basic arguments $A_{1}, \ldots, A_{n}$ is increasing whenever $n$ increases, and larger than the maximum of the strengths of its parts $A_{i}$. 
Yanal uses $f(x, y)=x+y-x y$ for two independent convergent arguments with strengths $x, y$ (Yanal, 1991, p. 140), which satisfies this principle. If $x, y>0$ then $f(x, y)>x$ and $f(x, y)>y$, and moreover $f(x, 0)=x$ and $f(0, y)=y$. The function is also symmetric $f(x, y)=f(y, x)$ and associative $f(f(x, y), z)=f(x, f(y, z))$, so it can be used for a larger number of converging arguments without introducing an undesirable order-dependence. Selinger defends a variation $g(x, y)=2 x+2 y-2 x y-1$ and requires that $x, y>\frac{1}{2}$ (Selinger, 2014, p. 386). For such inputs, the function satisfies ACU. However, in Selinger's approach a very bad additional argument (e.g. $y=0.2)$ decreases the overall strength of the argument such that it may fall below the threshold. For example, $g(0.6,0.2)=0.36$. From a psychological point of view this might appear to be reasonable, but it seems hard to justify from an ideally rational perspective that an acceptable argument $\left(x>\frac{1}{2}\right)$ becomes unacceptable by merely mentioning it together with another argument that is unacceptable. I thefore reject Selinger's rule in favor of Yanal's. ${ }^{3}$

\subsection{The Strength of Linked Arguments}

The idea behind a linked argument is that all required premises need to work together to support the conclusion. One criterion for determining whether an argument is linked or not is whether a subset of the premises could support an inference for or against the conclusion on their own. If a subset can itself form an argument, then the argument as a whole is not linked, but constitutes at least two convergent arguments. If, on the other hand, no proper subset of the linked premises can for itself form a pro or contra argument for the conclusion, but the totality of linked premises does constitute an acceptable argument, then the argument is linked. This characterization of linked arguments is rejected by Freeman (2011), cf. the discussion in Yanal (2002), and I do not claim that it is unproblematic. The criterion only works for those premises that are required for the conclusion to go through, but there might be reasons to allow additional premises that form a thematic unity with the rest of the argument. For this reason, I regard the criterion as a useful rule of thumb for cases when the required premises can be identified easily rather than as a definite criterion. Another difference to convergent arguments is that in a linked argument one missing premise will render the argument inconclusive or at least highly contentious. Together these two rules of thumb suffice to distinguish linked from convergent arguments.

As a typical example, in a deductive inference from $(p \wedge q) \rightarrow r, p$, $q$, to $r, p$ and $q$ are linked premises, in this case premises that are linked 
by logical conjunction, and no subset of $\{p, q,(p \wedge q) \rightarrow r\}$ alone serves as a deductively valid argument for $r$. This in turn means that if one of the premises is not sound, then the argument as a whole cannot be sound either. Consequently, if we consider soundness in terms of degree of acceptability, then the least strong premise must determine the strength of the argument as a whole. ${ }^{4}$

\section{Exclusionary Approach}

The following principle makes the above considerations explicit:

\section{(EML) Missing Linked Premise Principle}

(a) The strength of a linked argument that misses one or more required premises is below or at some lower threshold.

(b) The lower threshold cannot be higher than the strength of the weakest premise.

Linked arguments have a number $a_{1}, \ldots, a_{n}$ of required premises, and for some uses of bipolar argumentation graphs it may be desirable to annotate the number of required arguments directly in the scheme. As we have seen from the above deductive example, whenever one of the required premises is missing, the strength of the whole argument is significantly weakened. If the lower threshold is above zero, then the second part of the principle is needed to ensure that leaving out a very weak required premise cannot make the argument stronger, as this would be highly counter-intuitive in combination with the next principle and is generally not reconcilable with the idea that the premises are required.

Note that the lower threshold is not global, it is not independent of the initial assessment, because it not only depends on the number of required premises, but also on their strengths in a given application of the scheme. This complication can be avoided by setting the threshold to zero, and this practice is consistent with the general ideas behind an exclusionary account. Generally, presuming a zero lower threshold seems harder to defend for an ampliative approach, though, and in both approaches only makes sense if a distinction is made between premises that are left out, because they are known to be unsupported, and those that are left implicit in enthymemes. In the first case, the missing premise can be treated just like a required premise with minimal strength. In the second case, a zero lower threshold can become highly counter-intuitive, since it is very common to leave out required premises in practice. However, it can be equally counter-intuitive to allow a strength at or below a non-zero lower thresh- 


\section{Erich Rast}

old for an enthymematic linked argument when the missing premise can be trivially completed and is uncontroversial. Always taking missing required premises of linked arguments as a reason to assign a strength at or below the lower threshold is the least charitable assessment and therefore not adequate in general.

A reasonable way to deal with this issue is to give up EML and only consider subgraphs with all required premises. If the missing premise of a linked argument is completed in this way, but assigned a very low strength, then the following principle alone suffices for the exclusionary approach. ${ }^{5}$

\section{(EWL) Weakest Link Principle}

The strength of a linked argument is the minimum strength of its required premises.

This principle is well-known from possibility theory. ${ }^{6}$ In the present setting, it follows directly from the definition of a linked argument given above. The strength of a premise in a linked argument must be somehow connected to its credibility with respect to its role in the linked argument, and the same considerations as for chains apply. If the premise with the lowest credibility turns out false, then the strength of the overall argument falls down to or below the lower threshold no matter how strong the other premises are. Hence, the overall strength cannot exceed the minimum strength of the premises in an exclusionary account in which there is no interplay between argument quantity and quality.

Note that in the above example there must be a degree of support from $p$ to $r$, from $q$ to $r$ and from $(p \wedge q) \rightarrow r$ to $r$. However, it is clear that none of these assessments can be taken in isolation, as in a convergent argument, in this case, but that they instead have to be taken with regard to the linked argument as a whole, since otherwise the formal premise $(p \wedge q) \rightarrow r$ ought not give any credence at all to $r$ and the individual premises $p$ and $q$ alone could also only boost $r$ 's credence under some probabilistic or abductive reinterpretation. So, for the purpose of determining combinative argument strength, the premises of a linked argument cannot be regarded in isolation, since otherwise we would end up with a trivial theory that simply assigns strength 1 to any logically valid argument and 0 to any logically invalid argument.

\section{Ampliative Approach}

How does a corresponding ampliative principle for linked argument look? Such a principle will have to take into account thresholds in one form 
or another, too. The following principle is suitable, but not the only possible candidate:

\section{(ALT) Ampliative Linked Argument}

The strength of a linked argument that has $k$ premises and requires at least $n$ premises is equal to or above an upper threshold $U \geq 0.5$ for $k \geq n$ premises and equal to or below a lower threshold $L<0.5$ for $k<n$ premises.

This suggests a range of $k$-ary threshold functions $S_{n}^{k}\left(x_{1}, \ldots, x_{k}\right)$ such that for $k \geq n, S_{n}^{k}\left(x_{1}, \ldots, x_{k}\right) \geq U$ and for $k<n, S_{n}^{k}\left(x_{1}, \ldots, x_{k}\right) \leq L$ close to zero. The functions can be increasing with increasing $k(k \geq n)$ and decreasing with decreasing $k(k<n)$. From this characterization it is clear that ALT does not uniquely define a mode of combination, and further justification is needed for any particular way in which the functions are supposed to increase or decrease. In addition to ALT we could use an ampliative mode of combination in accordance with ACU such as Yanal's Rule whenever $k \geq n$. Alternatively, the functions may just yield the thresholds themselves. The simplest case is when $U=1, L=0$, and for a set of premises $\left\{a_{1}, \ldots, a_{n}\right\}$ and a conclusion $b$ in a logical language $L$ characterized by inference relation $\vdash_{L}$ the function yields 1 if $k=n, a_{1}, \ldots, a_{k} \vdash_{L} b$ and $\left\{a_{1}, \ldots, a_{k}\right\}$ are L-consistent, 0 otherwise. However, this approach would not be very useful, because it entirely disregards the initial assessment of the strength of the premises with respect to the inference step $x_{1}, \ldots x_{n}$. Other than such negative constraints, it is difficult to find any convincing arguments for characterizations of linked argument strength that are more specific than ALT. The reason for this difficulty seems to be that the problem of ampliative argument strength combination is underdetermined (see Section 4).

\subsection{The Strength of Argument Chains}

The four different types of chains between two arguments were listed in Figure 5 of Section 2. In a bipolar setting in which there are pro and contra arguments for a standpoint, the chains starting with a pro argument need to be treated differently from the chains starting with a contra argument. In an ampliative approach a pro antecedent will reinforce the strength of the succedent argument. In contrast to this, a very strong contra argument at the beginning of a chain will weaken the strength of the following argument. So principles for (a) and (b) need to differ from principles for (c) and (d) in Figure 5. 


\section{Exclusionary Approach}

The justification of the following principle mirrors that for EWL:

\section{(EWP) Weakest Pro Principle}

The strength of a pro-contra chain or a chain consisting of only pro links is the minimum of all strengths of the links in the chain.

According to Dubois \& Prade, this principle can be traced back to Theophrastus and is a basic principle of possibilistic logic (Dubois \& Prade 2009b: 2244). According to Selinger (2014), the Polish logician Tokarz (2006) also defends it for models of argumentation.

In a chain of inferences in which each prior standpoint acts as a reason (viz., justification in linguistic form) of the following standpoint or conclusion, and in which all of them are either pro or contra, but not mixed, each inference except for the first one depends on a previous inference, and the weaker the previous inference is, the weaker will be the overall conclusion, since each inference is a condition for the next inference to obtain. So, the strength of the reasoning chain as a whole can also not be higher than the strength of the weakest inference link. At the same time there is no good reason to assume that the overall inference strength declines independently of the strengths of the individual links.

Cayrol \& Lagasquie-Schiex give the example of an evaluation with negative argument strength for arguments with negative polarity (Cayrol \& Lagasquie-Schiex 2009: 79). In my view the phrase 'negative strength' makes no sense, though, and it is better to keep polarity and strength conceptually apart. Therefore, the principle also holds for pro-contra chains with a scheme as in Figure 5b, because contra arguments have positive strength. However, as laid out above, the principle cannot hold for contra chains. Take a mixed chain consisting of a contra argument from $a$ to $b$ with initial strength $x$ and a pro argument from $b$ to $c$ with initial strength $y$, for example. In this scenario, if $x$ is low, then $y$ must be high, since the first argument is against $b$; the overall strength of a chained argument should be high in the absence of other pro and contra arguments if the only attack of the pro argument is weak. The following general principles reflect these considerations:

\section{(EGP) General Attack/Support Principle}

If $S\left(x_{1}, x_{2}, \ldots, x_{n}\right)$ is the function for the combined strength of a mixed chain $x_{1}, \ldots, x_{n}$, then $S$ is increasing in each pro argument $x_{i}$ and decreasing in each contra argument $x_{j}$. 
The principle makes explicit what is meant by 'pro' and 'contra'. It is a combination of principles DA and BQ. The procedure outlined in Section 3.8 will take this principle into account. As it stands, EGP is far too liberal, allowing all kinds of counter-intuitive increases and decreases of argument strength in a chain. Additional principles are needed in order to fix appropriate upper and lower limits:

\section{$(\mathrm{EC}+)$ Contra Chain Maximum}

The combined strength of a contra argument $C_{1}$ directly followed by a pro (contra) argument $C_{2}$ cannot be higher than the strength of $C_{2}$.

Since a contra argument speaks against a standpoint, any chain of reasoning that is attacked by such an argument cannot become stronger than it would be without an attack. However, an attack might have no strength at all, and so the maximum strength of the chain under attack is the one that it had if it had not been attacked at all, i.e., its initial assessment if no other attackers or supporters are present. This holds for both pro and contra arguments as the second part of the chain, since the strength of contra arguments is also positive.

\section{(EC-) Contra Chain Minimum}

The combined strength of a contra argument $C_{1}$ directly followed by a pro (contra) argument $C_{2}$ is minimal whenever the strength of $C_{1}$ is maximal.

Recall that the initial assessment is supposed to represent an evaluation of the strength of arguments considered in isolation. Hence, an attack with maximum strength completely defeats the argument it attacks and so the resulting strength of the chain is minimal. This holds for both pro and contra arguments as the second part of the chain, since the strength of contra arguments is also positive. I therefore suggest that EGP, EC+ and ECare necessary principles for any viable account of the strength of argument chains in a bipolar setting. As in the previous cases, they do not uniquely determine a function for contra-chains, but in the absence of further criteria EC+ and EC- strongly suggest $S(x, y)=y-x y$ as a simple solution for contra chains in Figure 5cd when a measure of strength is defined over the unit interval $[0,1]$. As the strength of the antecedent contra argument approaches 1 , the strength of the successor argument should approach 0 , and, vice versa, as the strength of the antecedent contra argument approaches 0 , the strength of the successor argument should approach the level of its initial assessment. If another function is used, for instance one that introduces 
a nonlinearity, then additional criteria must be found to justify this more complicated choice, for $y-x y$ is the simplest non-trivial way of satisfying EGP, EC+, and EC-.

\section{Ampliative Approach}

We can accept EGP, EC+ and EC- for the reasons laid out above, but replace EWP by a more general principle:

\section{(APC) Ampliative Pro Chain}

The combined strength of a pro argument $A$ directly followed by a pro or contra argument $B$ goes against the minimum as the strength of $A$ approaches the minimum, and against the strength of $B$ as the strength of $A$ approaches the maximum.

Multiplicative combination of pro argument chains satisfies APC and makes sense if each argument is independent of each other and if one treats the dependencies between arguments in a pro chain as a conjunction. In possibility theory, both the minimum operation and multiplication are t-norms and, thus, can be regarded as generalizations of logical conjunction, ${ }^{7}$ and multiplication is a natural choice for a t-norm in an ampliative setting.

Which t-norm is chosen again seems to hinge on additional criteria and it is difficult to find any decisive argument for one norm or another. Generally speaking, ampliative bipolar theories that allow for contra chains are underdeveloped. Selinger's approach is unipolar. Cayrol \& Lagasquie-Schiex give a general characterization (Cayrol \& Lagasquie-Schiex 2009: 78/9), but the two examples of evaluation functions they provide are not further motivated. Related work in possibility theory such as Dubois \& Prade (2005) looks promising, but does not translate directly to argumentation graphs. That being said, EGP, EC+ and EC- are adequate for an ampliative approach to the strength of contra chains, too, for the same reasons as outlined above, and so $S(x, y)=y-x y$ also appears to be adequate for these types of theories.

\subsection{Link Attackers and Link Supporters}

Let us now turn to link attackers and supporters. An example of link support would be an argument like: "That conclusion isn't just likely, according to our current knowledge it follows almost with certainty." Link attacks are even more common, since any kind of doubt raised about the inference pattern is a link attack. For example, the following are link at- 
tacks: "That's a non-sequitur!", "That's a fallacy!", and "I'm not sure. This conclusion is maybe suggested by the data, but the sample size is fairly small."

Although there can be clear-cut cases if proponent and opponent agree on a shared methodology, generally speaking, attacking and supporting inference links are more problematic than the previous cases, because it is not at all clear what an uncertain inference is and there are many rival approaches to the modelling of uncertain inference. ${ }^{8}$ Since a link attack or support must somehow decrease or increase the credibility of an inference in an argument, it is a fortiori hard to justify particular modes of argument strength combination for these types of indirect arguments. In the following paragraphs I will discuss some principles that are intuitively plausible and (partially) justifiable in the current bipolar setting.

\section{Exclusionary Approach}

Although this is ultimately unsatisfactory, I suggest treating link attack and support like argument chains in an exclusionary approach. The reason why this is unsatisfactory is that there are strong intuitions towards taking link attackers and supporters as diminishing and augmenting the strength of an argument, which suggests ampliative principles. If ampliativity is flatout rejected in a strict exclusionary approach, then all we are left with is the following principle:

\section{(ELS) Link Support Principle}

The strength of an argument with a link supporter with initial strength $x$ for a link between $A$ and $b$ with strength $y$ is the minimum of $x$ and $y$.

Similarly to EWP, the idea behind this principle is that an argument can only have its full strength if its inference step is fully supported. So if link support is regarded in isolation to the rest of the argument, and if the strength of the argument is $y$ without considering link support, then it can remain $y$ only if the link is supported with its maximum value, i.e., when $x=1$ in an approach with argument strength in $[0,1]$. Moreover, from the limited exclusionary point of view, an argument can never be stronger than the strength of its inference step or the strength of its premises taken in isolation. Unsound premises do not become 'more sound' by arguing for the acceptability of the inference step, and an already unacceptable inference step does not become acceptable by making the premises stronger. Take for instance any classical logical fallacy. If the inference is invalid to start with, 


\section{Erich Rast}

then increasing the soundness of one or more premises will not make the argument stronger overall, because the inference to the conclusion will remain just as invalid as before. Hence, under the exclusionary understanding of link support, the inference cannot be stronger than this support and, therefore, the argument as a whole can also not be stronger than that support. This justifies ELS, though only under the given exclusionary interpretation which can be counter-intuitive to people who naturally lean towards ampliative principles.

Similar considerations lead to the following principle for link attacks:

\section{(ELA) Link Attack Principle}

The strength of an argument with link attacker with initial strength $x$ for a link between $A$ and $b$ with strength $y$ is going against $y$ as $x$ goes against the minimum, and against the minimum as $x$ goes against the maximum.

This is a variant of EC+ and EC- for link attackers and the justification is analogous to the above one for link support. If an attack on an inference step is completely pointless, i.e. has minimum strength, then the attacked argument ought not be diminished in strength at all. If, on the other hand, an attack on an inference step is maximally strong, then this means that the inference step is wholly unacceptable, and an argument based on an unacceptable inference cannot be acceptable, no matter how appealing the premises may be.

\section{Ampliative Approach}

Note that there are two possible interpretations of link attackers and supporters in the current setting in which a prior strength is associated with arguments. First, attackers and supporters may be taken akin to argument chains, except that they pertain to the inference rather than premises. Second, they could be understood as providing additional support or casting additional doubt on the strength of the inference. In the first case, all considerations of Section 3.4 apply, so the strength of an argument with prior strength and link support should be $x y$ and the strength with a link attack should be $y-x y$, as in chains. Generally speaking, link attackers and supporters impose additional conditions on overall argument strength in this view, which constitutes an ampliative counterpart to ELS. In the second case, additional support is modelled on the basis of ACU as in Section 3.2, using Yanal's ampliative combination $x+y-x y$, whereas attacks work the same as in the first case. 


\subsection{Mixed Bundles and Multiple Argumentation}

To recall, as a 'mixed bundle' we have characterized a number of competing pro and contra arguments for and against some standpoint, whereas multiple argumentation is a case of either multiple pro or multiple contra arguments against a standpoint, but not both pro and contra arguments. How to deal with such cases? I'd like to suggest principles similar to those that govern the combination of argument strength within linked and convergent arguments, but as I will lay out below, they need not necessarily be the same.

\section{Exclusionary Approach}

One may call a theory that endorses the following principle noncompensatory:

\section{(EMB) Maximum Bundle Principle}

The strongest pro or contra argument uniquely determines the strength of a mixed bundle and multiple arguments in case of multiple argumentation; other arguments have no effect.

This property is radical but has been defended in the literature on Practical Reasoning by authors like Horty, who reject the weighing conception of practical reasons (Horty 2012: 3-5). From this perspective, either a pro or contra argument is decisive or it is not. If it is decisive, then other arguments with the same polarity are redundant, and parallel counter-arguments are regarded as defeated or outranked. If it is not decisive, then either another argument in the mixed bundle is decisive or the argument as a whole is inconclusive. Horty allows for the latter possibility (Horty 2012: 47-53), and the next principle also endorses it.

\section{(ERC) Reason Conflict Principle}

In a bundle $a$ in which the strongest pro argument is as strong as the strongest contra argument, the strength of $a$ is at the minimum.

If the strongest pro and contra arguments are equally acceptable, then the bundle as a whole cannot be acceptable. From an exclusionary point of view, such an argument is not conclusive, so no conclusion should be drawn from it.

\section{Ampliative Approach}

To satisfy ampliativity, convergent bundles $a_{1}, \ldots, a_{n}$ of polarity $p$ on $b$ should yield an overall $p$-argument with a strength that is larger than the 
maximum strength $\operatorname{Max}\left(x_{i}\right)$ of the strengths $x_{1}, \ldots, x_{n}$ of the links from $a_{i}$ to $b$, which suggests using the same function as discussed in Section 3.2.9

\section{(ABP) Ampliative Bundle Principle}

Bundles of the same polarity are treated like complex convergent arguments.

The theory may additionally be compensatory, which is reflected by the following principle:

\section{(ACO) Compensatory Bundle Principle}

The total strength of all pro bundles on $C$ is weighed against the total strength of all contra bundles on $C$ by a function that (i) is increasing in its first argument for the pro bundle and (ii) decreasing in its second argument for the contra bundle.

Łukasiewicz's t-norm $\operatorname{Max}(0, x+y-1)$ adjusted for negative polarity as $S(x, y)=\operatorname{Max}(0, x-y)$ fulfills this requirement and represents a sceptical weighing conception. A less cautious weighing conception is given, as in the above mode, for contra chains as $S^{\prime}(x, y)=x-x y$. On the other hand, $f(x, y)=-x y+1$ is not acceptable, since $f(a, 0)=1$ even if $a$ is close to zero. Of course, an account can also be ampliative without being compensatory; in that case, the strength of a convergent argument with a given polarity satisfies ampliativity while at the same time EMB and maybe also ERC are accepted for the mixed case.

As for choosing a particular function, as in the previous examples, additional justifications would have to be given, but at the time of this writing no decisive arguments for a set of criteria that yields a unique solution to the problem are available. The problem of ampliative argument strength for bundles appears to be underdetermined.

\subsection{Conductive Arguments}

Conductive arguments combine several premises that mutually reinforce each other, but, in contrast to linked arguments, they are not substantially weakened when one of the premises is taken away. According to Govier, conductive arguments are a subspecies of convergent arguments, but they also allow for counter considerations (Govier 2013: 353; 355-366). They allow for mixed cases in our terminology, too, but, as a terminological choice, I do not classify them as convergent in order to distinguish the two types in the framework more easily; it would, of course, also be possible to introduce different types of convergent arguments instead. 
The following statement is a typical example of a conductive argument: "London is a great place to live despite high rents and high transportation costs; there are so many attractions, beautiful buildings, museums and things to do, and the city is bursting with life." It consists of several pro and some additional contra considerations, and the overall conclusion is that the pro considerations outweigh the contra considerations, which is the reason why these arguments are also sometimes called 'balance of consideration arguments' (Govier 2013: 353). It is striking that in the city example various criteria are combined in some way to support a conclusion, that these types of arguments seem to play a crucial role in practical and evaluative reasoning, and that there is therefore a close relation of these arguments to research in multi-criteria decision making like Fishburn (1970) and Keeney \& Raiffa (1976).

\section{Exclusionary Approach}

Since conductive arguments are based on some way of weighing pro against contra considerations, the exclusionary account reaches its limits with them. They are ampliative by definition. If one had to force an exclusionary evaluation of strength for them, it would have to be the same as for a convergent argument that beats another convergent argument of the opposite polarity. However, this approach seems too coarse-grained to be of any practical relevance.

\section{Ampliative Approach}

On the one hand, an ampliative approach to conductive arguments must differ from linked arguments, as their strength does not fall down to a threshold when a premise is taken away as in the case of a linked argument. In contrast to mixed bundles, on the other hand, subarguments or premises of the same polarity do not first have to be combined, before weighing them against all arguments of the opposite polarity, or at least some additional justification would be needed to justify this particular mode of aggregation. The following principle reflects these characteristics, where premises are taken to be ordered into two groups according to their polarity:

\section{(ACA) Ampliative Conductive Argument}

In a conductive argument the strengths of the pro premises $x_{1}, \ldots, x_{k}$ is weighed against the strengths of the contra premises $x_{k+1}, \ldots, x_{n}$ by a function $F\left(x_{1}, \ldots, x_{k} ; x_{k+1}, \ldots, x_{n}\right)$ that is increasing in $x_{1}, \ldots, x_{k}$ and decreasing in $x_{k+1}, \ldots, x_{n}$. If $p$ is pro (contra), then $F$ approaches its maximum as $x_{1}, \ldots, x_{k}$ approach their maximum (minimum) val- 
ues and $x_{k+1}, \ldots, x_{n}$ approach their minimum (maximum) values, and $F$ approaches its minimum as $x_{1}, \ldots, x_{k}$ approach their minimum (maximum) values and $x_{k+1}, \ldots, x_{n}$ approach their maximum (minimum) values.

Let $w_{i}=1$ if $p_{i}=\oplus, w_{i}=-1$ otherwise. Then the normalized sum fulfills ACA:

$$
F\left(x_{1}, \ldots, x_{n}\right)=\frac{1}{n} \sum_{i=1}^{n} w_{i} x_{i}
$$

However, there is a huge problem with this way of thinking about conductive arguments that needs to be mentioned, even though I cannot go into the details here. It is, in my view, incorrect to mix up argument strength with the evaluative contribution of reasons, since (i) an argument for and support for a certain level of a cardinal or ordinal value attribute such as 'quality of life' or 'costs' can be more or less strong, (ii) there may be different types of value or criteria both formally (e.g. additive vs. lexicographic) and substantially (e.g. different qualities of value in value pluralism), but there is only one degree of argument strength, (iii) it is known from multi-attribute decision making that numerical representations of multiple criteria that are ordered by preferences must satisfy corresponding representation theorems. ${ }^{10}$ Consequently, the normalized sum can only be used to represent argument strength in conductive arguments without further ado, and it seems that many types of conductive arguments need to additionally take into account explicit representations of the evaluative component, which must satisfy corresponding representation theorems in order to make the argument acceptable - if a representation theorem is violated, the argument strength as a whole needs to fall to a lower threshold as in a linked argument. More work is needed to elaborate the distinction between argument strength and evaluative components of conductive arguments. ${ }^{11}$

\subsection{Recursive Valuation Procedure}

In this section, an example will illustrate the combination of the principles discussed so far. I will not give a strict definition of how to go through a BAG to assign particular values, as it is easier to illustrate this procedure by example. The principal idea is very straightforward: In the absence of cycles, we have to traverse the graph from its initial nodes to the terminal nodes, determine the structure of the (sub-)arguments in question, and choose a particular mode of argument strength combination that satisfies 
the principles discussed in the previous sections. As I have laid out above, in some cases exactly one function is at least strongly suggested. Most of the time, however, the concrete functions that were given are only candidates that in the absence of further constraints are plausible.

\section{Exclusionary Approach}

Evaluation starts at the initial leaves of the BAG. These are the premises of one of the above argument types, and an initial valuation is associated either with each premise or with the links. To each conclusion $b$, one of the above modes of combination is applied in order as follows: (1) The strength of a linked argument is the minimum strength of the strength of its premises. (2) The strength of a basic argument is its initial strength. (3) The strength of a convergent argument consisting of basic or linked antecedents $A_{1}, A_{2}, \ldots, A_{n}$ is the maximum of the strengths of $A_{1}, A_{2}, \ldots, A_{n}$. (4) The strength $y$ of one of (1)-(3) plus a link supporter with strength $x$ is $\operatorname{Min}(x, y)$. (5) The strength $y$ of one of (1)-(4) plus a link attack with strength $x$ is $y-x y$. (6) If the argument(s) for or against $b$ form the first part of a chain to $c$, then the strength of the chain to $c$ is calculated as described in Section 3.2 by nesting of two-argument rules in the direction of argument flow. (7) The strength of a mixed bundle is calculated as outlined above. (8) The strength of a larger graph is calculated by repeatedly applying steps (1)-(7) in the direction of argument flow until all terminal nodes have been reached. The end result of the antecedent to the terminal node is the strength of the conclusion at that node.

\section{Example valuation:}

Figure 6 depicts a bipolar argument graph and the steps needed for its recursive evaluation: $\alpha$. Convergent pro argument $S(\alpha)=0.9 ; \beta$. Linked contra argument $S(\beta)=\operatorname{Min}(0.5,0.7)=0.5 ; \gamma$. link attack on $\beta$ with $S(\gamma)=\operatorname{Min}(0.7,0.5) ; \delta$. mixed bundle on $d$ where $\alpha$ beats $\gamma$ and so $S(\delta)=$ $S(\alpha)=0.9 ; \epsilon$. pro-pro chain on $e$ with $S(\epsilon)=\operatorname{Min}(0.9,0.8)=0.8$, which is the final strength of $e$. Suppose instead that $\alpha$ was a convergent contra argument against $d$. Then it would still beat $\gamma$, but the final result would be $S(\epsilon)=0.8-0.9 \times 0.8=0.08$, i.e., the conclusion $e$ would have almost no argumentative support.

\section{Ampliative Approach}

This is like the exclusionary approach, except that corresponding ampliative principles are used and additionally conductive arguments are taken into account. 


\section{Example valuation:}

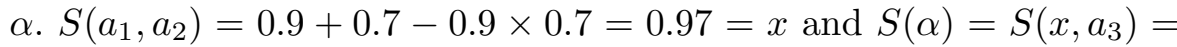
0.979 by Yanal's Rule. For the linked argument, assuming that all required premises are present and Yanal's Rule can be used, $S(\beta)=0.5+0.7-0.5 \times$ $0.7=0.85 . S(\gamma)=0.85-0.7 \times 0.85=0.255$ according to $S(x, y)=y-x y$. Assuming ABP, ACO, and Yanal's Rule again, $S(\delta)=0.979-0.979 \times$ $0.255=0.729355$. Finally, $S(\epsilon)=0.729355 \times 0.8=0.583484$. Notice that we still have to decide in the last step, whether the pro bundle beats the contra bundle in order to determine whether the chain is a pro-pro or contra-pro chain. Suppose, as in the previous example, that $\alpha$ was a convergent contra argument against $d$. We would then get an ampliative contra-bundle with $S(\delta)=0.85+0.255-0.85 \times 0.255=0.88825$ and a final contra-pro chain with $S(\epsilon)=0.8-0.88825 \times 0.8=0.0894$, i.e., almost the same result as in the previous case.

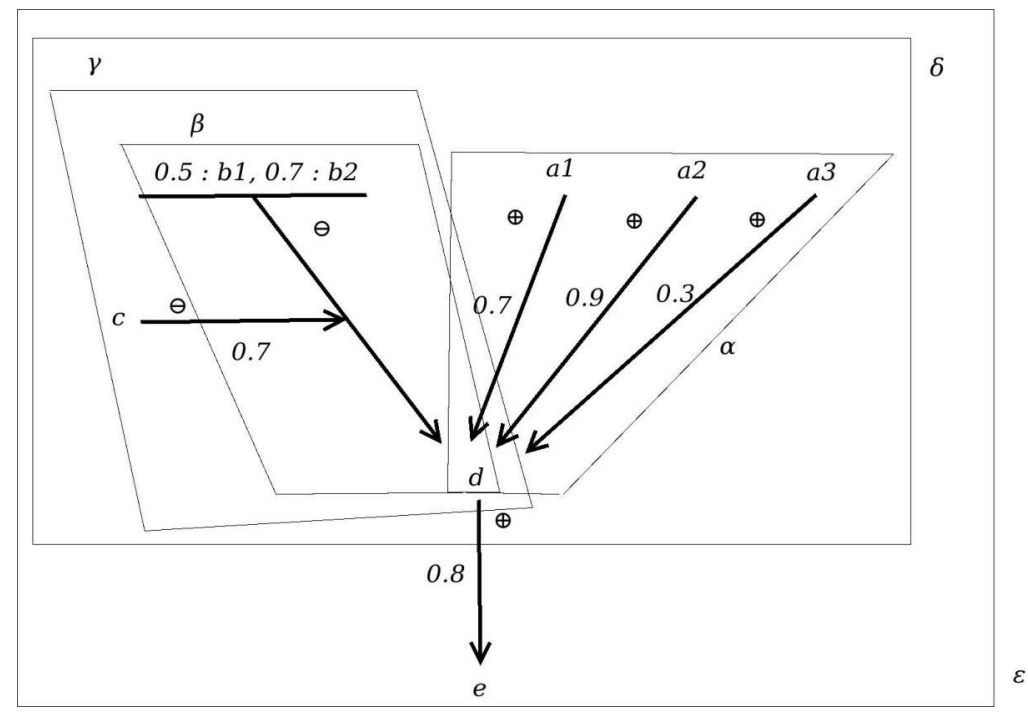

Figure 6. Example of a Bipolar Argumentation Graph with 5 evaluation steps $\alpha-\epsilon$. The argument in the graph starts with a convergent pro argument $\alpha$ and a linked contra argument $\beta$ that is in turn attacked by a link attack $\gamma$. The pro and contra arguments together form a mixed bundle argument $\delta$ for standpoint $d$, which in turn forms the premise of a pro argument $\epsilon$ for standpoint $e$. Opponent and proponent roles are not assigned in this type of model. 


\section{Critical Discussion}

We have seen exclusionary and ampliative rationality principles for the combination of argument strength, and in each case I have briefly justified why these are adequate for the combination of intuitive prior assessments of argument strength. However, a number of problems have become apparent from the previous sections that I would like to address in the remainder of this article.

\subsection{Problem Underdetermination}

A problem formulation can be overdetermined or underdetermined. If it is overdetermined, then the problem might not have a solution. If it is underdetermined, then there might be too many solutions or it might not even be possible to identify a reasonable range of solutions. As the discussion so far has shown, the problem of finding an intuitively compelling notion of combinative argument strength is underdetermined. Reasonable principles for ampliative and exclusionary argument strength do not determine functions for strength combination uniquely. Instead, the range of admissible functions is infinite and the suggested modes of combination were chosen on the basis of simplicity. To obtain specific solutions, for example a set of necessary and sufficient axioms, additional principles would be needed. Where could these come from? It seems to me that additional principles would need to come from the rules that govern the particular type of argument-whether it is deductive, inductive, or abductive.

To illustrate this point, let us take another look at the independence requirements for the premises of linked and convergent arguments. Suppose we have two seemingly convergent applications of modus ponens in deductive reasoning such that $\{a, a \rightarrow c\}$ forms one basic argument and $\{b, a \rightarrow c\}$ another basic argument for conclusion $c$. If in this example additionally $a \rightarrow b$ held, then the second argument would be redundant, hence rules like EP and ACU would not be applicable. These principles can only apply if the two premises are logically independent of each other, or, as a heuristic, if their logical relation to each other is not known. If, contrary to that, $a \rightarrow b$ is known, then a rational assessment of $b$ 's stength cannot intuitively be higher than that of $a$. It is evident that in a probabilistic framework a corresponding independence condition would be $P(A \mid B)=P(A)$ or some more complex condition in a more elaborate probabilistic setting such as Jøsang (2008), and yet another formulation of independence will be needed for a particular account of abduction. The idea behind rules like EP is that the arguments cannot be spelt out in all detail with precise numbers available, but that 


\section{Erich Rast}

it still makes sense to assume or presume that the premises are sufficiently independent of each other. But this is an assumption, and if more is known about the type of argument at play and whether the corresponding independence condition is fulfilled or not, then rules laid out in the previous section will yield incorrect results. They are underdetermined in order to allow the intuitive assessment of the strength of arguments of very different types without going into the details, and in that respect only serve as heuristics.

There is a more fundamental problem with conductive arguments. As laid out above, balance of consideration type arguments are based on evaluations which have multiple aspects or dimensions. These can be more or less acceptable, hence the strength of considerations for or against a standpoint may vary independently of the evaluative component 'value vs. disvalue of a certain kind'. The strengths of the considerations is what (loosely) corresponds to the weighting of different criteria in multi-criteria decision making, but from the point of view of argumentation theory the standard model of decision making makes too many assumptions. A strong consideration for or against some standpoint in a conductive argument may also lexicographically outrank other considerations, for instance, and there are many different ways to combine multiple criteria qualitatively or quantitatively. Argument strength for conductive arguments is intertwined with the problem of determining multiple criteria, aspects of betterness, or value dimensions and how to rationally aggregate them into overall assessments. So, the problem of combinative argument strength for conductive arguments is additionally underdetermined, because the notion of conductive argument is itself unclear and problematic. If individual aspects of values in such arguments could generally be represented by utilities and strength could be represented by weights and probability measures, then expected utility in multi-attribute utility theory could be used to combine them into an overall assessment of a certain 'strength'. However, this approach is inadequate for qualitative (ordinal) value aggregation and not general enough to deal with all types of conductive arguments. Unless this problem is solved, conductive arguments and their relation to non-traditional and traditional multi-attribute decision making remain unclear and consequently the role of (sub-)argument strengths (aka weights in decision making) in balance of consideration arguments will remain unclear.

\subsection{Overdetermination}

There is another problem. The rules discussed in the previous sections sometimes also overdetermine combinative argument strength, because they 
sometimes give the wrong result. This is easiest to see with ampliativity (AP). Of course, ampliativity cannot be literally a property of every argument. Consider any convergent pro argument or multiple pro arguments for the same standpoint. If the arguments are deductive, then additional premises may either be redundant or they might contradict each other, in case of which the argument may become trivial. ${ }^{12}$ As laid out above, ampliativity can only hold for logically independent premises. But things are even worse for abductive and probabilistic reasoning patterns, as these are nonmonotonic and this property means that additional premises that seem to count in favour of the conclusion can actually make the conclusion less credible. So AP cannot hold in general, even though it may serve as a useful heuristic in many cases.

By the same token, it is also possible to construct counter-examples to corresponding exclusionary principles. For example, if a convergent argument is, in the end, upon closer scrutiny probabilistic, then additional evidence that is spelt out in the form of additional premises may indeed raise the level of support for the conclusion. In abduction this is even the canonical case: Additional indicators often strengthen the degree of plausibility of the most plausible conclusion in comparison to other conclusions. So, principle EP is not only information lossy, it may under certain circumstances defeat the whole purpose of arguing in a certain way.

At the same time, the fact that such principles appear to be intuitively plausible indicates that they may serve as useful heuristics and constitute some weak form of 'ecological rationality'. In other words, they are not irrational, but they do not constitute strict rationality principles in the sense of being generally truth-conducive.

\subsection{The Connection to Epistemology}

I would like to suggest that the problems characterized in the previous paragraphs are based on a deeper issue. Aside from further dialectic criteria, argument strength primarily pertains to the 'quality' of the premises and inference step, but (i) the quality of the premises hinges on the available evidence and thus, ultimately, on the state of nature or on mathematical laws, and (ii) the quality of the inference step hinges on the type of inference and maybe some considerations about how likely it is that the inference was correct or successful in practice. Because of the second dependence, a fully justified theory of argument strength would need to use different principles for different types of inference, such as deduction, induction, abduction, and value-based conductive arguments in practical reasoning. It is clear, for instance, that from an ideally rational perspective a deductive inference 


\section{Erich Rast}

can only be fully acceptable when it is logically valid and in practice an assessment of the probability that an error has been made would be needed in order to get a justified 'degree of strength' for such an inference. Likewise, inductive inferences must meet the correctness criteria of proper inferential statistics and it has been mentioned above that at least some types of value representations in some conductive arguments have to satisfy representation theorems of multi-criteria decision making. All of these differences lead to different criteria, and so it seems that no uniform theory is possible.

Because of the first dependence, rational theories of argument strength are split into two branches. An agent-relative theory assesses the quality of premises on the basis of an agent's epistemic state. However, it may be argued convincingly that such a theory would not be a theory of argument strength in the general sense, since the strength of an argument can hardly vary from person to person; it would be a theory of perceived argument strength, and then one may ask why such a theory ought not be a purely psychological theory of how convincing humans find arguments. In contrast to this, an agent-agnostic theory evaluates the quality of premises on the basis of the state of nature in addition to logical and mathematical principles. But this is nothing other than what is studied in the philosophy of science and parts of epistemology! Although, perhaps, ideally rationally justifiable, such a theory does not capture an intuitive notion of argument strength, but rather lays out a general epistemological framework. There are also reasonable doubts whether such a theory is feasible in general, since there are many different methods of inquiry and theory formation and there does not seem to be any 'logic of scientific discovery'. Be that as it may, a fully comprehensive theory that links arguments to evidence and methods of inquiry would have to make far too many and too strong ideal rationality requirements to be useful as a theory of argument strength in argumentative practice. That is the conundrum faced by any theory of argument strength.

\section{Conclusion}

Basic principles for the combination of argument strength in complex arguments in a bipolar setting have been laid out and partially justified. I have then argued that these cannot serve as general rationality postulates, despite their intuitive appeal, and instead should rather be regarded as reflecting heuristics that may sometimes be useful and sometimes might fail.

However, this observation does not preclude more specific approaches to argument strength that are obtained by taking into account more specific 
inference patterns, theories of uncertain inference, and notions of support by premises. For example, the above principles can be refined in terms of possibility theory, probability theory, or Dempster-Shafer theory. My main complaint about such approaches is one of practicality. The more precise these theories are made, the more the endeavour as a whole becomes one of laying out a general epistemological framework, a generic method of inquiry as some Bayesians have attempted, and the less practical it therefore becomes as a general theory of argument strength. I believe that an exclusionary theory like the one presented in this article may serve as a reasonable and cautious middle ground between a purely psychological account and a theoretically sound epistemology that, nevertheless, remains of merely theoretical interest, because in practice data is often too sparse and too imprecise to elaborate all the details of an argument.

\section{N O T E S}

1 See Hitchcock (2015), cf. Yanal (2002, 20-3) and Freeman (2011, Ch. 5). To make things worse, as Hitchcock sets out, Govier's notion of conductive arguments seems to overlap with ways in which convergent arguments have been understood, and neither of these distinctions matches the pragma-dialectic distinction between coordinatively compound and multiple arguments (Hitchcock, 2015, 4-6). See also Snoeck Henkemans (2000) who gives an overview of similar, though not identical distinctions made in Pragma-Dialectic Argumentation Theory.

2 Selinger (2014) makes the same requirements. Besnard \& Hunter (2008) make the assumption that argument graphs must be trees, which is stronger than what is needed here.

${ }^{3}$ Following a suggestion by an anonymous reviewer, it is worth mentioning in this context that a bad argument for $C$ does not generally make a good argument for not $C$. By comparison, in probability calculus $P(\operatorname{not} A)=1-P(A)$. Such a principle would be inadequate for argument strength.

4 These considerations are only acceptable under a material reading of the inference according to which $p$ and $q$ contribute positive reasons for $r$ in light of the major premise, because they are acceptable as true. There is also a formal reading according to which the logical support of the premises is maximal if $p$ and $q$ are false and $(p \wedge q) \rightarrow r$ is considered fully acceptable. The difference is between logical and epistemic support, and strictly speaking we ought not use the truth-functional conditional ' $\rightarrow$ ' for a reading that excludes the second case. However, there is no need to enter this well-known debate on natural language vs. logical conditionals here; the example is simplified for illustration.

5 This practice is admissible, since a linked argument can only be recognized as such if missing premises are identified. The same does not hold for other types of arguments, except basic arguments with just one premise. In fact, it is not even clear what would make a conductive or a convergent argument enthymematic in the first place.

6 See for instance Dubois (2009b).

7 See Haehnle (2001, 301); cf. Dubois \& Prade $(1988,2004)$ for introductions.

8 See Kyburg \& Teng (2001). 
9 A theory that does not satisfy this criterion is nonampliative. For example, averaging the strengths is nonampliative.

10 Point (iii) follows from results in measurement theory such as Debreu (1954), Krantz et al (1971), Roberts (1979), whereas theories without numerical representations have to find reasonable ways of evading variants of Arrow's Theorem like those discussed in Bouyssou \& Pirlot (2003). Point (i) is similar to the combination of quantified risk with utility in Multi-attribute Utility Theory: Risk enters evaluation by a principle such as the Expected Utility Hypothesis, for instance, but it is not the same as the evaluative component, and a similar relationship must hold for argument strength and an evaluative component.

11 There are reasons for being sceptical that the above structures for conductive arguments can faithfully represent value- and criteria-based reasoning, as they cannot easily be used to represent the essentially comparative nature of that reasoning. For example, in practical reasoning, meaningful comparisons between different attributes are only possible when the maximum and minimum levels of those attributes are known to the decision maker (Eisenfuehr et al. 2010: 151-154). This topic is left for another occasion.

12 Whether ex falso quod libet constitutes a problem or not depends on many factors and I wish to avoid this discussion. What counts here is the AP will no longer hold, or is at least very hard to justify for those cases.

\section{R E F E R E N C E S}

Adler, J. (2013). Are conductive arguments possible? Argumentation, 27(3): 245257.

Besnard, P. and Hunter, A. (2008). Elements of Argumentation. MIT Press, Cambridge MA.

Blair, A. J. and Johnson, R. H., editors (2011). Conductive Argument: An Overlooked Type of Defeasible Reasoning, London. College Publications.

Bouyssou, D. and Pirlot, M. (2003). Ordinal aggregation and strict preferences for multi-attributed alternatives. Internal Report Cahier du LAMSADE 212, Université Paris Dauphine, Paris, France.

Cayrol, C. and Lagasquie-Schiex, M. C. (2005). Gradual valuation in bipolar argumentation frameworks. In Godo, L., editor, Symbolic and Quantitative Approaches to Reasoning with Uncertainty, pages 366-377, Berlin, Heidelberg. Springer.

Cayrol, C. and Lagasquie-Schiex, M. C. (2009). Bipolar abstract argumentation systems. In Rahwan, I. and Simari, G. R., editors, Argumentation in Artificial Intelligence, pages 65-84. Springer.

Debreu, G. (1954). Representation of preference ordering by a numerical function. In Thrall, R., Coombs, C., and Davies, R., editors, Decision Processes, pages 159-175. Wiley, New York.

Dubois, D. and Prade, H. (1988). Possibility Theory. Springer, New York.

Dubois, D. and Prade, H. (2004). Possibilistic logic: A retrospective and prospective view. Fuzzy Sets and Systems, 144:3-23. 
Dubois, D. and Prade, H. (2005). A bipolar possibilistic representation of knowledge and preferences and its applications. In Isabelle Bloch, Alfredo Petrosino, A. T., editor, Fuzzy logic and applications: 6th international workshop, WILF 2005, Crema, Italy, September 15-17, 2005: revised selected papers, pages 1-10. Springer.

Dubois, D. and Prade, H. (2009). Possibility theory. In Meyers, R. A., editor, Computational Complexity: Theory, Techniques, and Applications, pages 22402252. Springer.

Dung, P. M. (1995). On the acceptability of arguments and its fundamental role in nonmonotonic reasoning, logic programming and n-person games. Artificial Intelligence, $77(2): 321-357$.

Eisenführ, F., Weber, M., and Langer, T. (2010). Rational Decision Making. Springer.

Fishburn, P. C. (1970). Utility Theory for Decision Making. John Wiley and Sons, New York, London, Sidney, Toronto.

Freeman, J. B. (2011). Argument Structure. Springer, Dordrecht Heidelberg London New York.

Govier, T. (2013). A Practical Study of Argument. Wadsworth CENGAGE Learning, Boston, enhanced 7th edition edition.

Hähnle, R. (2001). Advanced many-valued logics. In Gabbay, D. M. and Guenthner, F., editors, Handbook of Philosophical Logic, Vol. 2, volume 2, pages 297-395. Springer.

Hitchcock, D. (1983). Critical Thinking: A Guide to Evaluating Information. Methuen, Toronto.

Hitchcock, D. (2015). The linked-covergent distinction. In van Eemeren, F. H. and Garssen, B., editors, Reflections on Theoretical Issues in Argumentation Theory, pages 83-91. Springer.

Hitchcock, D. (1980). Deduction, induction and conduction. Informal Logic Newsletter, 3:7-15.

Horty, J. F. (2012). Reasons as Defaults. Cambridge University Press, Oxford/New York.

Jøsang, A. (2008). Conditional Reasoning with Subjective Logic. Journal of Multiple-Valued Logic and Soft Computing, Vol. 15, No. 1, pages 5-38.

Jin, R. (2011). The structure of pro and con arguments: A survey of the theories. In Blair, A., editor, Conductive Argument, pages 10-30. College Publications.

Keeney, R. L. and Raiffa, H. (1976). Decisions with Multiple Objectives: Preferences and Value Tradeoffs. John Wiley \& Sons, New York.

Krantz, D. H., Luce, R. D., Suppes, P., and Tversky (1971, 1989, 1990). Foundations of Measurement, Volumes I-III. Academic Press, New York.

Kyburg, H. E., Jr., and Teng, C. M. (2001). Uncertain Inference. Cambridge UP. 
Nielsen, S. and Parsons, S. (2006). An application of formal argumentation: Fusing Bayes nets in multi-agent systems. In Proceedings of the First Conference on Computational Models of Argument, pages 33-44, Amsterdam. IOS Press.

Roberts, F. S. (1979). Measurement Theory. Adison Wesley, Reading, MA.

Selinger, M. (2014). Towards formal representation and evaluation of arguments. Argumentation, 28:379-393.

Snoeck Henkemans, F. A. (2000). State-of-the-art: The structure of argumentation. Argumentation, 14:447-473.

Thomas, S. N. (1977). Practical Reasoning in Natural Language. Prentice-Hall, Upper Saddle River, NJ.

Tokarz, M. (2006). Argumentacja, perswazja, manipulacja [Argumentation, persuasion, manipulation]. Gdańskie Wydawnictwo Psychologiczne, Gdańsk.

Walton, D. (1996). Argument Structure: A Pragmatic Theory. University of Toronto Press, Toronto.

Wellman, C. (1971). Challenge and Response: Justification in Ethics. Southern Illinois University Press, Carbondale, IL.

Wellman, C. (1975). Morals and Ethics. Prentice Hall, Englewood Cliffs, NJ.

Yanal, R. J. (1991). Dependent and independent reasons. Informal Logic, XIII(3): $137-144$.

Yanal, R. J. (2003). Linked and convergent reasons - again. In Blair, A., Johnson, R. H., Hansen, H. V., and Tindale, C. W., editors, Proceedings of the Ontario Society for the Study of Argumentation Conference, Vol. 5, pages 17, Windsor, Canada. University of Windsor, University of Windsor.

Zenker, F. (2011). Deduction, induction, conduction: An attempt at unifying natural language argument structures. In Blair, A. J. and Johnson, R. H., editors, Conductive Argument: An Overlooked Type of Defeasible Reasoning, pages 74-85. College Publications. 